ORIGINAL ARTICLE

\section{High serum concentrations of lipopolysaccharide binding protein in pregnancies with pre-eclampsia}

\author{
Yoshiko Sakabe ${ }^{1,2}$, Haruki Nishizawa ${ }^{1}$, Asuka Kato ${ }^{2}$, \\ Hikari Yoshizawa $^{1,2}$, Yoshiteru Noda ${ }^{1,2}$, Akiko Ohwaki ${ }^{1,2}$, \\ Takao Sekiya ${ }^{1}$, Takuma Fujii ${ }^{1}$, Hiroki Kurahashi ${ }^{2}$ \\ ${ }^{1}$ Department of Obstetrics and Gynecology, Fujita Health University School of Medicine, \\ Toyoake, Japan, ${ }^{2}$ Division of Molecular Genetics, Institute for Comprehensive Medical \\ Science, Fujita Health University, Toyoake, Japan
}

Reprint request to:

Haruki Nishizawa, M.D., Ph.D., Department of Obstetrics and Gynecology, Fujita Health University, 1-98 Dengakugakubo, Kutsukake, Toyoake, Aichi 4701192, Japan.

E-mail:nharuki@fujita-hu.ac.jp

\section{Key words:}

lipopolysaccharide binding protein, microbiome, pre-eclampsia

Received: July 7, 2021

Revised: August 13, 2021

Accepted: August 16, 2021

J-STAGE Advance published date: September 17, 2021

DOI:10.14390/jsshp.HRP2021-013

\begin{abstract}
Aim: Pre-eclampsia is considered a multi-factorial disease, in which both fetal and maternal factors are involved in its onset. In this study, we aimed to test the longstanding hypothesis that the immune response to bacterial endotoxin could explain the etiology of pre-eclampsia.

Methods: Serum lipopolysaccharide binding protein (LBP) concentrations and the vaginal microbiome were compared between subjects with pre-eclampsia and those with normotensive pregnancies.

Results: Serum LBP concentrations were higher in subjects with pre-eclampsia compared to those with normotensive pregnancies. LBP concentrations were poorly correlated with disease onset, blood pressure, birthweight, and placental weight. Moreover, there was no evidence of bacterial species specific to pre-eclampsia based on a comparative analysis of the vaginal microbiome of subjects with pre-eclampsia and those with normotensive pregnancies.

Conclusion: Serum LBP concentrations were significantly higher in subjects with pre-eclampsia compared to those with normotensive pregnancies. The clinical significance of high serum LBP concentrations in pre-eclampsia requires further investigation.
\end{abstract}

\section{Introduction}

Pre-eclampsia is principally defined by the onset of hypertension with or without proteinuria, ${ }^{1,2)}$ and is one of the most common obstetric complications, accounting for almost $15 \%$ of pregnancy-associated disorders. Preeclampsia is a multiple organ failure syndrome involving the liver, kidneys, and lungs, in addition to coagulation and neural system difficulties. It is far more complex than pregnancy induced hypertension (PIH), and has a higher rate of maternal mortality compared to other obstetric complications. It is well-documented that preeclampsia is a multi-factorial disease that is triggered by many genetic and environmental factors. ${ }^{3-5}$ ) With regard to maternal factors, the possible involvement of bacterial infection in the etiology of pre-eclampsia is a longstanding, unproven hypothesis. ${ }^{6,7)}$

Lipopolysaccharide binding protein (LBP) is an endogenous host protein that mediates interactions between bacterial endotoxin, i.e., lipopolysaccharide, and toll-like receptor 4 on immune competent cells. This association requires the aid of $\mathrm{CD} 14$ protein to activate the downstream signal cascade for innate immunity. ${ }^{8)}$ LBP is mainly produced as an acute phase protein by hepatocytes during infection, and its concentration increases following exposure to gram-negative bacteria. Increased concentrations of LBP result in the activation of macrophages and monocytes to control the infection. A number of studies have demonstrated the clinical usefulness of LBP in plasma or amniotic fluid as a disease biomarker of various obstetric complications, such as preterm labor and premature rupture of the membranes. ${ }^{9,10)}$ Notably, estimating LBP concentrations may help discriminate between bacterial infection and systemic inflammatory response syndrome. ${ }^{11)}$ To investigate the possible involvement of bacterial 
lipopolysaccharide binding protein in pre-eclampsia

Table 1. Characteristics of the study subjects with a normotensive pregnancy and with pre-eclampsia

\begin{tabular}{|c|c|c|c|c|c|c|}
\hline & $\begin{array}{c}\text { Normotensive } \\
\text { pregnancy }\end{array}$ & $\begin{array}{c}\text { Early onset } \\
\text { Pre-eclampsia }\end{array}$ & $\begin{array}{c}\text { Late onset } \\
\text { Pre-eclampsia }\end{array}$ & & $P$ Value & \\
\hline & $n=48$ & $n=19$ & $n=23$ & $\begin{array}{l}\text { NP vs } \\
\text { EO-PE }\end{array}$ & $\begin{array}{l}\text { NP vs } \\
\text { LO-PE }\end{array}$ & $\begin{array}{c}\text { EO-PE vS } \\
\text { LO-PE }\end{array}$ \\
\hline Maternal age (y) & $31.4 \pm 4.6^{\dagger}$ & $31.7 \pm 5.2$ & $30.8 \pm 4.4$ & n.s & n.s & n.s \\
\hline Gestational age (weeks) & $35.1 \pm 4.2$ & $30.1 \pm 2.2$ & $36.0 \pm 1.7$ & $<0.05$ & n.s & $<0.05$ \\
\hline Systolic BP (mmHg) & $112.6 \pm 10.2$ & $165.8 \pm 15.4$ & $161.6 \pm 9.0$ & $<0.05$ & $<0.05$ & n.s \\
\hline Diastolic BP (mmHg) & $67.4 \pm 8.2$ & $102.1 \pm 14.2$ & $100.4 \pm 9.7$ & $<0.05$ & $<0.05$ & n.s \\
\hline Proteinuria $^{\ddagger}$ & $0(0 \%)$ & $19(100 \%)$ & $23(100 \%)$ & $<0.05$ & $<0.05$ & n.s \\
\hline Body mass index $(\mathrm{BMI})^{\S}$ & $20.9 \pm 3.0$ & $22.1 \pm 3.7$ & $21.9 \pm 4.3$ & n.s & n.s & n.s \\
\hline Birth weight (g) & $2,984.7 \pm 350.1$ & $1,109.6 \pm 312.9$ & $2,013.8 \pm 472.8$ & $<0.05$ & $<0.05$ & $<0.05$ \\
\hline Birth weight coefficient & $1.011 \pm 0.129$ & $0.686 \pm 0.119$ & $0.793 \pm 0.131$ & $<0.05$ & $<0.05$ & $<0.05$ \\
\hline Placental weight (g) & $576.4 \pm 88.4$ & $252.6 \pm 59.1$ & $380.9 \pm 88.7$ & $<0.05$ & $<0.05$ & $<0.05$ \\
\hline Placental weight coefficient & $1.035 \pm 0.166$ & $0.596 \pm 0.132$ & $0.719 \pm 0.157$ & $<0.05$ & $<0.05$ & $<0.05$ \\
\hline
\end{tabular}

infection in the etiology of pre-eclampsia, we measured serum LBP concentrations in affected women, evaluated pre-eclampsia-specific bacterial patterns by analyzing the vaginal microbiome of these women, and assessed whether LBP has clinical utility as a biomarker of preeclampsia.

\section{Materials and methods}

\section{Subjects}

All clinical samples analyzed in this study were collected at the Department of Obstetrics and Gynecology, Fujita Health University Hospital, Japan, from 2005 to 2014. Maternal blood samples were obtained during cesarean sections from subjects with severe pre-eclampsia $(n=$ 42), and from control subjects with uncomplicated normotensive pregnancies $(n=48)$. Pre-eclampsia was defined as a blood pressure of greater than 160/110 $\mathrm{mmHg}$, and by proteinuria of more than $2 \mathrm{~g}$ in a 24 $\mathrm{h}$ collection, although these criteria were revised and the latest version is currently used. ${ }^{2,12)}$ We excluded other types of PIH, such as gestational hypertension, superimposed pre-eclampsia, eclampsia, and mild PIH, from the analysis. Normotensive subjects were matched with subjects with pre-eclampsia for maternal age and body mass index (BMI) during pre-pregnancy. The clinical details of these subjects are presented in Table 1. We calculated the birthweight coefficient by dividing the measured birthweight by the expected standard birthweight at the gestational week. ${ }^{13)}$ We also calculated the placental weight coefficient by dividing the measured placental weight by the expected standard placental weight at the gestational week. ${ }^{14)}$ Vaginal swab samples from subjects with pre-eclampsia were obtained at admission. Samples were also obtained from women with normal uncomplicated pregnancies that were matched for the gestational week of the pre-eclampsia specimens. Serum and swab samples were stored at $-80^{\circ} \mathrm{C}$ until use. Informed consent was obtained from each subject. This study was approved by the Fujita Health University Ethics Review Committee for Clinical Studies.

\section{Enzyme-linked immunosorbent assay (ELISA)}

Serum LBP concentrations were measured using a commercially available LBP soluble ELISA kit (Enzo Life Sciences, Farmingdale, NY) in accordance with the manufacturer's instructions. A dilution series of recombinant human LBP was used to establish standard curves, and all samples were run in duplicate. The intra- and inter-assay coefficients of variation were less than $9.3 \%$ and $8.1 \%$, respectively. The detection limit for recombinant LBP was approximately $4 \mu \mathrm{g} / \mathrm{ml}$. The calibration curve was linear up to $40 \mu \mathrm{g} / \mathrm{ml}$.

PCR amplification and sequencing of 16S rRNA gene V3-4 regions

DNA was isolated from vaginal wall swabs using a QIAamp DNA Microbiome Kit (QIAGEN GmbH, Hilden, Germany) in accordance with the manufacturer's instructions. DNA samples were stored at $20^{\circ} \mathrm{C}$ until use. DNA amplification was conducted using the following universal primers: forward (5'-TCGTCGGCAGCGTCA GATGTGTATAAGAGACAGTCGTCGGCAGCGTCA GATGTGTATAAGAGACAGCCTACGGGNGGCWGC AG-3') and reverse (-5'-GTCTCGTGGGCTCGGAG ATGTGTATAAGAGACAGGTCTCGTGGGCTCGGA GATGTGTATAAGAGACAGGACTACHVGGGTATCT AATCC -3') to target the V3-4 regions of bacterial $16 \mathrm{~S}$ 
rRNA. The bold letters denote the Illumina overhang adapter. Individual samples were barcoded, pooled to construct the sequencing library, and then sequenced using Illumina Miseq (Illumina, San Diego, CA) to generate pair-ended $300 \times 300$ reads.

\section{Statistical analysis}

Intergroup comparisons were performed using the MannWhitney $U$ test. $P$ values less than 0.05 were considered statistically significant. Correlations were evaluated using Spearman's test. In significant difference tests, $P$ values were calculated with the $\mathrm{z}$ conversion of Fisher's r. $P$ values less than 0.05 were considered statistically significant for these analyses as well. A beta-diversity measure, the weighted UniFrac distance matrix which measures the pairwise difference in microbial diversity among samples, was calculated using QIIME. To provide visualization of sample distribution patterns, principal component analysis (PCA) was applied to transform the UniFrac distance matrices into principal coordinates.

\section{Results}

Serum LBP concentrations were higher in the preeclampsia study cohort compared with a control population of subjects with normotensive pregnancies (Figure 1). As early onset pre-eclampsia is often associated with more severe symptoms, the pre-eclampsia cohort was divided into two groups: 19 early onset cases (earlier than 34 weeks gestation) and 23 late onset cases (34 weeks gestation or later). No significant difference in LBP concentrations was observed between these two pre-eclampsia subgroups (Figure 1).

To further evaluate the utility of LBP as a biomarker, we examined the correlation between LBP concentrations and various clinical parameters. This analysis revealed a significant association between LBP concentrations and systolic and diastolic blood pressure (Figures 2A and 2B). Notably, however, this correlation was not observed when the analyses were performed separately for the pre-eclampsia group and normotensive group. This suggests that the correlation between LBP concentrations and blood pressure simply reflected the presence of the disease, rather than its severity. A similar observation was made when we evaluated the association between LBP concentrations and birthweight or placental weight. Specifically, serum LBP concentrations correlated negatively with normalized values for these two weight measures (Figures 2C and 2D), although again no correlation was observed when the analyses were performed separately for the pre-eclampsia group and normotensive group. No correlations were observed between LBP concentrations and other assessed clinical parameters, such as uric acid concentration, platelet

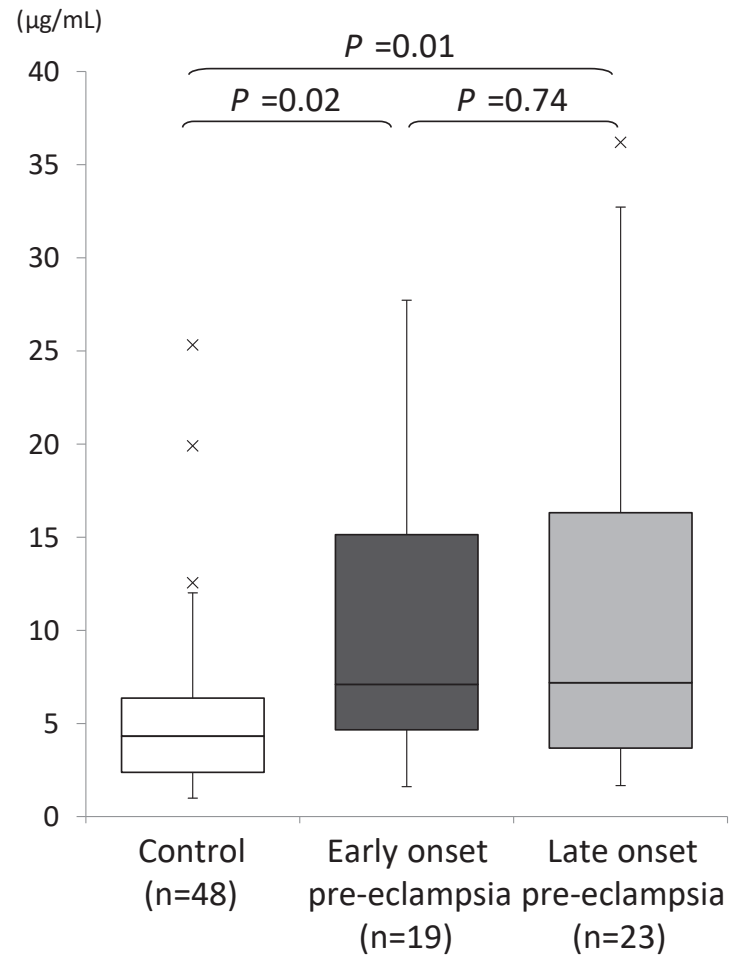

Figure 1. Serum LBP concentrations measured by ELISA. Serum LBP concentrations were compared between uncomplicated normotensive pregnancies (left), early onset ( $<34 w$ ) pre-eclampsia (middle), and late onset ( $>34 \mathrm{w}$ ) pre-eclampsia (right). The boxes indicate 25th and 75th percentiles, and the bands near the middle denote median values. Bars indicate 1.5 interquartile ranges with outliers specifically marked.

count, and quantified urinary protein (Supplementary Figure).

To test the hypothesis that increased LBP concentrations in the maternal circulation may reflect bacterial infection rather than systemic inflammatory response syndrome, we analyzed the vaginal microbiome of subjects in the pre-eclampsia and normotensive groups by deep sequencing of PCR amplicons of the V3-4 regions of the bacterial 16S rRNA gene. The DNA used in these amplification reactions was obtained from swab samples. A total of four samples from the pre-eclampsia group and 24 from the normotensive group were analyzed. PCA analysis was first conducted to determine whether the global microbiome profile of each sample could differentiate between the pre-eclampsia and normotensive groups. However, the pre-eclampsia samples did not cluster in this analysis (Figure 3). Next, the abundance of each bacterial strain was compared between samples of the two groups (Figure 4). However, none of the bacterial species were found to be specific for the pre-eclampsia group. 

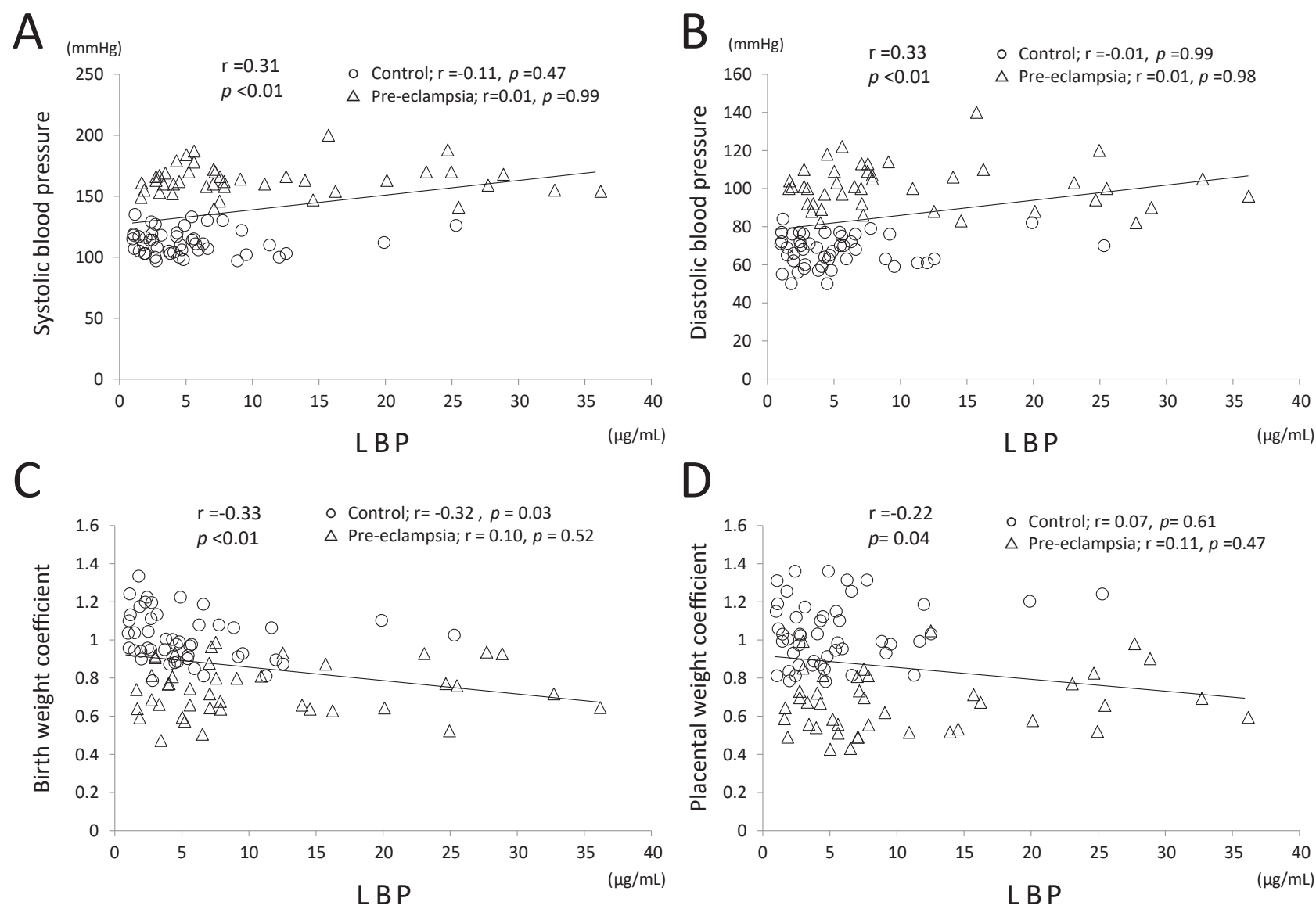

Figure 2. Correlations between serum LBP concentrations and systolic blood pressure (A), diastolic blood pressure (B), normalized birth weight (C), and normalized placental weight (D). Open circles indicate normotensive (uncomplicated) subjects and open triangles indicate pre-eclampsia subjects. Regression lines are shown with correlation coefficients and $\boldsymbol{P}$ values.

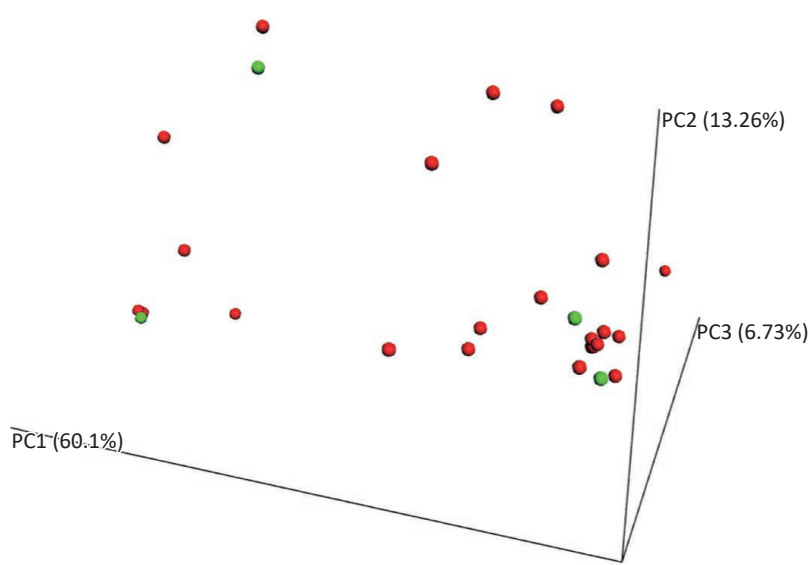

Figure 3. PCA analysis plot constructed using weighted UniFrac analysis. Red circles indicate normotensive subjects and green circles indicate pre-eclampsia subjects.

\section{Discussion}

This report is the first to describe high serum LBP concentrations in women with pre-eclampsia. It is generally understood that the levels of a considerable number of soluble factors are elevated in the maternal circulation in pre-eclampsia, most of which originate from aberrant increases in gene expression and protein production in the pre-eclamptic placenta. Notably, the $L B P$ gene is not among genes previously reported to be highly expressed in the placenta of pre-eclamptic patients relative to those with normotensive pregnancies. ${ }^{15,16)}$ The public RNA sequence database indicates that the liver is the only tissue that abundantly expresses the $L B P$ gene (HPA RNA-seq normal tissues, PRJEB4337). Elevated LBP concentrations in pre-eclampsia are thus unlikely to originate from the placenta, but rather from the liver due to a systemic inflammatory response to bacterial or nonbacterial infections.

The increase in concentrations of circulating LBP 


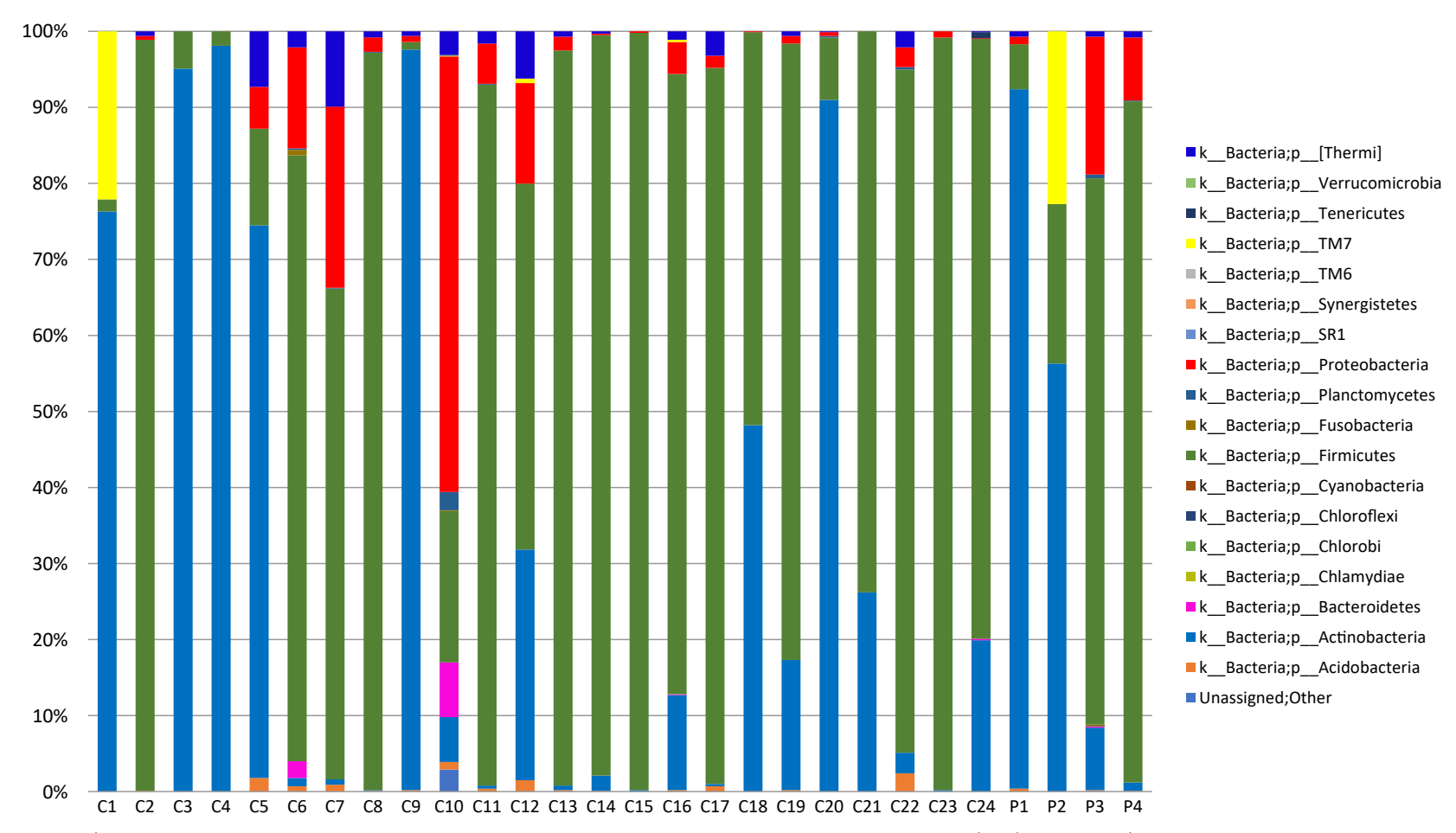

Control

Pre-eclampsia

Figure 4. Relative abundance of different bacterial phyla in each sample. Comparative analysis was performed between normotensive $(n=24)$ and pre-eclampsia $(n=4)$ subjects. The seven most abundant bacterial phyla are indicated.

in maternal serum in pre-eclampsia suggests the possible utility of LBP as a biomarker for this disorder. Circulating placental anti-angiogenic factors such as sFlt-1 or placental growth factor (PlGF) are known to predict the onset of pre-eclampsia. For instance, a recent study reported that the reduction of circulating PlGF is sufficiently sensitive to enable the pre-symptomatic diagnosis of pre-eclampsia. ${ }^{17}$ ) Serum LBP would be a useful biomarker if its concentrations in the serum increase before clinical onset of pre-eclampsia, which requires further investigation. To test whether LBP concentration is a marker for pre-eclampsia severity, we analyzed its association with known clinical parameters of the disorder. However, correlations between serum LBP concentrations and these parameters were only observed when samples from all pre-eclamptic subjects were analyzed together. This suggests that the observed correlation simply reflects the presence of the disease, but not its severity. Indeed, when we performed the analysis by dividing pre-eclamptic subjects into those with early onset or late onset, no correlations were observed between the pre-eclampsia subgroups and normotensive subjects. Thus, serum LBP concentration is not a predictor of preeclampsia severity.

The elevation of serum LBP concentrations in pre-eclampsia has been suggested to indicate the involvement of maternal bacterial infection. ${ }^{6,7)}$ Since bacterial endotoxin is considered the primary cause of pre-eclampsia, this condition is aptly referred to as pre-eclamptic toxemia of pregnancy. ${ }^{18)}$ Although it is now widely accepted that bacterial infection is not the principal etiological factor in pre-eclampsia, a dormant microbial component is thought to be involved in its onset. ${ }^{19)}$ Asymptomatic bacteria without an apparent urinary tract infection might be associated with preeclampsia. ${ }^{20)}$ Another hypothesis is that intra-abdominal hypertension gives rise to a translocation of intestinal bacterial endotoxins to the liver, thereby inducing systemic inflammation that leads to the symptoms of pre-eclampsia. ${ }^{18)}$ These possibilities led us to examine the vaginal microbiome in pre-eclampsia patients.

In a screen for infectious agents, the presence of pathogenic bacteria has been previously reported in the placental microbiome of pre-eclampsia patients. ${ }^{21)}$ However, a recent large-scale analysis found no evidence of bacteria in the placentas of pre-eclampsia cases. ${ }^{22)} \mathrm{A}$ report on the gut microbiome profile in pre-eclampsia indicated differences from a normal pregnancy and that this distinct profile returned to normal after birth. ${ }^{23)}$ Another study reported a gut microbiome profile that 
was specific to pre-eclampsia, and that oral inoculation of gut bacteria into pregnant mice induced pre-eclampsialike symptoms. ${ }^{24)}$ These data suggest that changes in the maternal microbiome may play a role in the development of pre-eclampsia and provide insight into mechanisms involved in the onset of this disorder. ${ }^{25,26)}$

Associations between the vaginal microbiome and specific bacteria and preterm births have been reported. ${ }^{27)}$ Although ample evidence exists that gut microbiota directly or indirectly plays a role in the pathogenesis of hypertension and pre-eclampsia, less information is available on the vaginal microbiome of women with pre-eclampsia. ${ }^{28-30)}$ We therefore focused on the vaginal microbiome in the present study, but did not find a preeclampsia-specific microbiome profile. However, one limitation of our study is the limited number of subjects, which may have made it difficult to detect significant differences. Another possibility is that high serum LBP concentrations do not indicate the presence of bacterial infection, but simply reflect systemic inflammatory response syndrome. ${ }^{11)}$ Although inflammatory cytokines, including acute phase proteins like C-reactive protein, are reportedly elevated in pre-eclampsia, it is still unclear whether this increase occurs before disease onset or is a consequence of the disease itself. ${ }^{7)}$ A more thorough investigation of the vaginal microbiome will be needed to robustly test the hypothesis that specific bacteria induce the development of pre-eclampsia.

In conclusion, we detected high serum concentrations of LBP in women with pre-eclampsia. However, it remains unclear whether maternal bacterial infection underlies the etiology of this disease. Understanding the clinical significance of high serum LBP concentrations in pre-eclampsia will require further investigation.

\section{Acknowledgements}

This study was supported by the Ogyaa Donation Foundation from the Japan Association of Obstetricians and Gynecologists, and by grants-in-aid for Scientific Research from the Ministry of Education, Culture, Sports, Science, and Technology, Japan (16K11117, 15H04710) and from the Ministry of Health, Labour and Welfare, Japan (H27-nanchitou (nan)-ippan-024).

\section{Conflict of Interest}

The authors declare no conflict of interest.

\section{References}

1. National Institutes of Health. Working group report on high blood pressure in pregnancy. Bethesda: NIH Publication no. 00-3029; 2000.
2. Takagi K, Yamasaki M, Nakamoto O, et al. A review of best practice guide 2015 for care and treatment of hypertension in pregnancy. Hypertens Res Pregnancy. 2015; 3: 65-103.

3. Roberts JM, Cooper DW. Pathogenesis and genetics of preeclampsia. Lancet. 2001; 357: 53-56.

4. Cross JC. The genetics of pre-eclampsia: a feto-placental or maternal problem? Clin Genet. 2003; 64: 96-103.

5. Sibai B, Dekker G, Kupferminc M. Pre-eclampsia. Lancet. 2005; 365: 785-799.

6. Conde-Agudelo A, Villar J, Lindheimer M. Maternal infection and risk of preeclampsia: systematic review and metaanalysis. Am J Obstet Gynecol. 2008; 198: 7-22.

7. Rustveld LO, Kelsey SF, Sharma R. Association between maternal infections and preeclampsia: a systematic review of epidemiologic studies. Matern Child Health J. 2008; 12: 223-242.

8. Schumann RR. Old and new findings on lipopolysaccharidebinding protein: a soluble pattern-recognition molecule. Biochem Soc Trans. 2011; 39: 989-993.

9. Gardella C, Hitti J, Martin TR, Ruzinski JT, Eschenbach D. Amniotic fluid lipopolysaccharide-binding protein and soluble CD14 as mediators of the inflammatory response in preterm labor. Am J Obstet Gynecol. 2001; 184: 1241-1248.

10. Chen FC, Sarioglu N, Büscher U, Dudenhausen JW. Lipopolysaccharide binding protein in the early diagnosis of intraamniotic infection of pregnant women with premature rupture of the membranes. J Perinat Med. 2009; 37: 135-139.

11. Pavcnik-Arnol M, Hojker S, Derganc M. Lipopolysaccharidebinding protein, lipopolysaccharide, and soluble CD14 in sepsis of critically ill neonates and children. Intensive Care Med. 2007; 33 : $1025-1032$.

12. Watanabe K, Matsubara K, Nakamoto $\mathrm{O}$, et al. Outline of the new definition and classification of "Hypertensive Disorders of Pregnancy (HDP)"; a revised JSSHP statement of 2005. 2018; 6: $33-37$.

13. Uehara R, Miura F, Itabashi K, Fujimura M, Nakamura Y. Distribution of birth weight for gestational age in Japanese infants delivered by cesarean section. J Epidemiol. 2011; 21: 217-222.

14. Ogawa M, Matsuda Y, Nakai A, Hayashi M, Sato S, Matsubara S. Standard curves of placental weight and fetal/placental weight ratio in Japanese population: difference according to the delivery mode, fetal sex, or maternal parity. Eur J Obstet Gynecol Reprod Biol. 2016; 206: 225-231.

15. Nishizawa H, Pryor-Koishi K, Kato T, Kowa H, Kurahashi H, Udagawa Y. Microarray analysis of differentially expressed fetal genes in placental tissue derived from early and late onset severe pre-eclampsia. Placenta. 2007; 28: 487-497.

16. Nishizawa $\mathrm{H}$, Ota $\mathrm{S}$, Suzuki M, et al. Comparative gene expression profiling of placentas from patients with severe pre-eclampsia and unexplained fetal growth restriction. Reprod Biol Endocrinol. 2011; 9: 107.

17. Duhig KE, Myers J, Seed PT, et al. Placental growth factor testing to assess women with suspected pre-eclampsia: a multicentre, pragmatic, stepped-wedge cluster-randomised controlled trial. Lancet. 2019; 393: 1807-1818.

18. Sawchuck DJ, Wittmann BK. Pre-eclampsia renamed and reframed: Intra-abdominal hypertension in pregnancy. Med Hypotheses. 2014; 83: 619-632.

19. Kell DB, Kenny LC. A Dormant Microbial Component in the Development of Preeclampsia. Front Med (Lausanne). 2016; 3: 60. 


\section{Y. Sakabe et al.}

20. Rezavand N, Veisi F, Zangane M, Amini R, Almasi A. Association between Asymptomatic Bacteriuria and Pre-Eclampsia. Glob J Health Sci. 2015; 8: 235-239.

21. Amarasekara R, Jayasekara RW, Senanayake H, Dissanayake VH. Microbiome of the placenta in pre-eclampsia supports the role of bacteria in the multifactorial cause of pre-eclampsia. J Obstet Gynaecol Res. 2015; 41: 662-669.

22. de Goffau MC, Lager S, Sovio U, et al. Human placenta has no microbiome but can contain potential pathogens. Nature. 2019; 572: 329-334.

23. Lv LJ, Li SH, Li SC, et al. Early-Onset Preeclampsia Is Associated With Gut Microbial Alterations in Antepartum and Postpartum Women. Front Cell Infect Microbiol. 2019; 9: 224.

24. Chen X, Li P, Liu M, et al. Gut dysbiosis induces the development of pre-eclampsia through bacterial translocation. Gut. 2020; 69: $513-522$.

25. Ahmadian E, Rahbar Saadat Y, Hosseiniyan Khatibi SM, et al.
Pre-Eclampsia: Microbiota possibly playing a role. Pharmacol Res. 2020; 155: 104692.

26. Beckers KF, Sones JL. Maternal microbiome and the hypertensive disorder of pregnancy, preeclampsia. Am J Physiol Heart Circ Physiol. 2020; 318: H1-H10.

27. Fettweis JM, Serrano MG, Brooks JP, et al. The vaginal microbiome and preterm birth. Nat Med. 2019; 25: 1012-1021.

28. Liu J, Yang H, Yin Z, et al. Remodeling of the gut microbiota and structural shifts in Preeclampsia patients in South China. Eur J Clin Microbiol Infect Dis. 2017; 36: 713-719.

29. Marques FZ, Mackay CR, Kaye DM. Beyond gut feelings: how the gut microbiota regulates blood pressure. Nat Rev Cardiol. 2018; 15: $20-32$.

30. Lin CY, Lin CY, Yeh YM, et al. Severe preeclampsia is associated with a higher relative abundance of Prevotella bivia in the vaginal microbiota. Sci Rep. 2020; 10: 18249.
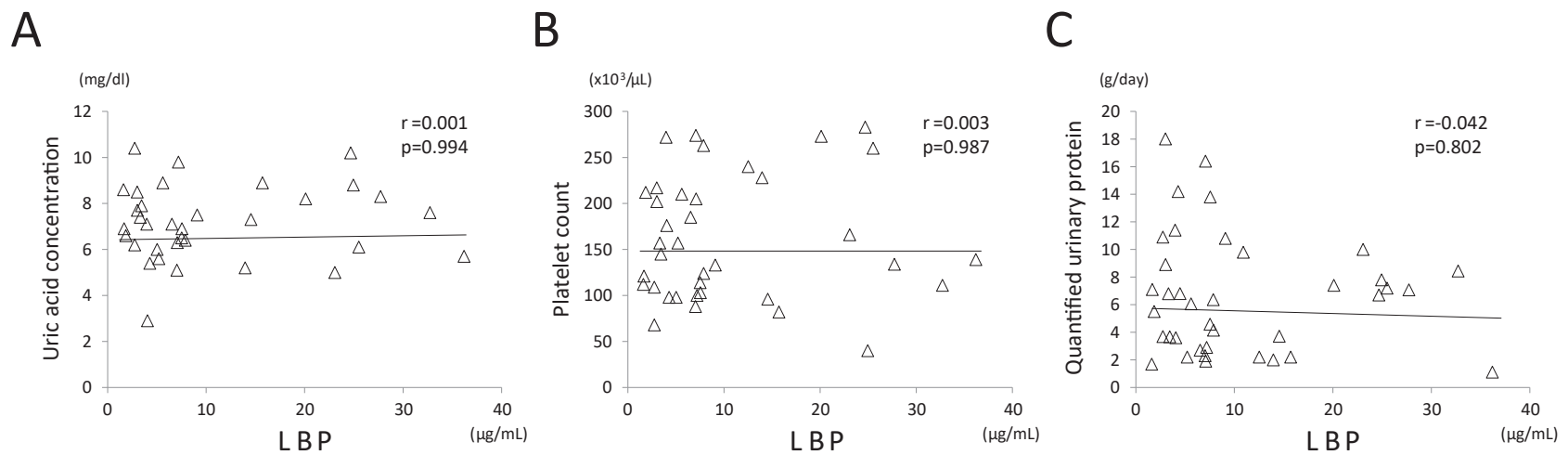

\section{Supplementary Figure. Correlations between serum LBP concentrations and uric acid concentration (A), platelet count (B), and quantified urinary protein (C). Only pre-eclampsia samples were analyzed (open triangles). Regression lines are shown with correlation coefficients and $\boldsymbol{P}$ values.}

